\title{
Uterine Corpus Carcinosarcoma
}

National Cancer Institute

\section{Source}

National Cancer Institute. Uterine Corpus Carcinosarcoma. NCI Thesaurus. Code C9180.

An aggressive malignant mixed epithelial and mesenchymal tumor that arises from the uterine corpus. It usually affects elderly postmenopausal women and presents with vaginal bleeding. It is characterized by the presence of a malignant epithelial component that is usually glandular and a sarcomatous component. 